\title{
Linx
}

Revue des linguistes de l'université Paris X Nanterre

9 | 1997

Émile Benveniste. Vingt ans après

\section{Benveniste : sémantique sans sémiotique}

\section{Henri Meschonnic}

\section{OpenEdition}

\section{Journals}

Édition électronique

URL : http://journals.openedition.org/linx/1075

DOI : 10.4000/linx.1075

ISSN : 2118-9692

\section{Éditeur}

Presses universitaires de Paris Nanterre

\section{Édition imprimée}

Date de publication : 1 avril 1997

Pagination : 307-326

ISSN : 0246-8743

\section{Référence électronique}

Henri Meschonnic, "Benveniste : sémantique sans sémiotique », Linx [En ligne], 9 | 1997, mis en ligne le 09 juillet 2012, consulté le 30 avril 2019. URL : http://journals.openedition.org/linx/1075 ; DOI :

10.4000/linx.1075 


\title{
Benveniste : sémantique sans sémiotique
}

\author{
Henri Meschonnic
}

$\mathrm{E}$ n trois mots, c'est toute la question de la poétique, à transformer en problème. C'est-à-dire à inventer. Par quoi la théorie du langage est à réinventer. Ce qui suppose entre la poétique et la théorie du langage une implication réciproque, une interaction, une réaction en chaîne.

Ici, je veux seulement montrer que Benveniste à la fois prépare cette implication théorique et (à ma connaissance, dans la limite des textes qu'on connaît de lui) ${ }^{1}$ ne la conçoit pas. Tout en se situant au point même où elle doit se faire.

Toute l'époque contemporaine, en ce qui concerne les relations entre le langage et l'art, y compris l'art du langage, peut se voir comme un conflit entre le côté de Peirce et le côté de Saussure, et surtout comme l'effacement ou plutôt la dénégation de ce conflit.

Ce qui est remarquable, dès le début de l'article de Benveniste "Sémiologie de la langue», d'où les trois mots sémantique sans sémiotique sont tirés, et qui est ici le point de départ, c'est que Benveniste, à mon sens, qu'il l'ait su ou non, pose le préalable de ce problème : « un grand problème a surgi qui n'a pas encore reçu sa forme précise $»^{2}$.

\footnotetext{
${ }^{1}$ Le colloque sur « Benveniste vingt ans après », en août 1995, aura en effet au moins rendu public ce fait notable, qu'il existe plusieurs centaines de pages de Benveniste sur la poétique en manuscrit et qu'il s'imposerait de publier sans plus attendre, sans faire attendre, abusivement, ce qu'on appelle la communauté scientifique.

2 Emile Benveniste, «Sémiologie de la langue » (1969), Problèmes de linguistique générale, Gallimard, 1974, tome II, p. 43. Ensuite, les références à cet article ne mentionnent que la page.
} 
Henri Meschonnic

Par là, un certain rapport de plusieurs linguistes contemporains à Benveniste $^{3}$ - sans compter l'ignorance des autres, particulièrement aux EtatsUnis - j'entends la relégation, et la condescendance, observables, sont autant de dénégations de ce "grand problème». Dénégation piquante pour des linguistes, car elle est la négation même de la notion de théorie du langage.

Ce qu'on peut constater, c'est qu'il y a plus de Peirce que de Saussure dans la pensée contemporaine. Saussure est doublement effacé, par Peirce, et par le structuralisme, par la vulgate structuraliste. Sauf sans doute chez ceux qui travaillent à l'histoire de la pensée du langage, ce qu'on ne saurait faire sans la pensée de cette pensée. Car, d'en faire l'histoire, ne peut consister à éviter de la penser. Il faut les deux, étant donné l'historicité de toute pensée. C'est le cas, chez Benveniste.

Benveniste, à la suite de Saussure, prend indissociablement l'histoire et la théorie, en posant qu'« une linguistique n'est possible qu'à cette condition : se connaître enfin en découvrant son objet » (p. 46).

C'est son propre inconnu que la théorie du langage essaie de reconnaître, en même temps que son objet. Aussitôt, ce que Benveniste dit de Saussure vaut pour lui-même, quand il parle de «termes qui créent leurs propres concepts» (p. 47).

Une démarche déductive. Chez tous les deux. Elle est la même, pour le passage sur la sémiologie, que cite Benveniste et où Saussure dit : «Puisqu'elle n'existe pas encore, on ne peut dire ce qu'elle sera; mais elle a droit à l'existence, sa place est déterminée d'avance ", et pour le $a$ long indo-européen dans le Mémoire de 1879. De même pour la «métasémantique » évoquée à la fin de «Sémiologie de la langue ».

C'est une démarche de reprise, de poursuite d'un même et autre objectif chez Benveniste: «Reprenant ce grand problème au point où Saussure l'a laissé [...]» (p. 50). Et moi aussi je dirais, toute proportion gardée, qu'il en est de même pour la poétique, telle que Benveniste lui prépare sa place : reprenant ce grand problème au point où Benveniste l'a laissé...

Je tenterai seulement de glisser ce qui est à penser en analysant quelques difficultés ou contradictions propres à la présentation de Benveniste, et qu'il ne porte pas, il me semble, à l'état de problèmes. Ce sont seulement ces quelques points que je cherche à porter à l'état de problèmes.

Pour commencer, Benveniste s'en tient à la définition traditionnelle du signe, comme «substitut » (p. 51). Alors que, vu de la poétique du rythme, le signe est cet ensemble de six paradigmes (linguistique, anthropologique, philosophique, théologique, social, politique), que j'analyse dans Politique $d u$ rythme, politique $d u$ sujet ${ }^{4}$. Pour Benveniste, le signe reste seulement

\footnotetext{
3 Je renvoie à mon analyse, «Seul comme Benveniste, ou Comment la critique manque de style», Langages $n^{\circ} 111$ juin 1995 .

4 Verdier, 1995.
} 
linguistique ${ }^{5}$ et, dans sa collecte initiale, avant ré-examen, il a un statut métaphorique (pour signal) ou sémiotique, dans la mention pêle-mêle des «'signes de politesse' », et des "signes de l'art dans leurs variétés (musique, images ; reproductions plastiques) $»(p .51)$.

Cet état commun à toutes les sortes de signes est le moment où Benveniste définit «leur propriété de signifier ou SIGNIFIANCE, et leur composition en unités de signifiance, ou SIGNES» (p. 51).Ce qui ne dit rien encore d'aucune propriété distinctive. Dont Benveniste lui-même, dans la suite de son article, montre la limitation, pour l'art.

Il prend le «système des feux du trafic routier» (p. 52) comme exemple d'un «modèle canonique du système binaire » (p. 53). Dont il dit que le mode opératoire visuel est "généralement diurne » (p. 52). Où j'observerais deux détails mineurs : les feux sont notoirement diurnes et nocturnes; et ils sont donnés pour une "opposition chromatique vert-rouge », en oubliant l'orange, mais il est vrai que c'est une phase préparatoire qui ne change rien à l'opposition fondamentalement binaire. Surtout il est curieux, sans qu'on sache jusque là pourquoi, que Benveniste n'emploie pas à ce propos le terme de signal, comme il faisait dans "Communication animale et langage humain », en 1952.

Aussitôt, ce qui éclaire la stratégie de l'emploi général du terme signe, Benveniste énonce un principe capital, en lettres capitales, le «PRINCIPE DE NON-REDONDANCE entre systèmes. Il n'y a pas de 'synonymie' entre systèmes sémiotiques ; on ne peut pas 'dire la même chose' par la parole et par la musique, qui sont des systèmes à base différente » (p. 53).

Cette «non-convertibilité entre systèmes à bases différentes» (p. 53) est la condition première du rapport entre la langue comme «l'interprétant de la société » (p. 54) et la « signifiance unidimensionnelle » (p. 65) des oeuvres d'art - sémantique sans sémiotique. C'est la condition du rapport entre le langage et la peinture, donc de ce qu'on peut dire d'une peinture ou d'une sculpture, mais aussi d'une oeuvre de langage. Ou plutôt de ce qu'on ne peut pas dire. Benveniste insiste sur l'effet, en art, de «monde clos» (p. 59). Curieusement, plus loin, la clôture est celle du signe : "En réalité le monde du signe est clos » (p. 65). C'est la clôture l'un à l'autre de «deux univers conceptuels» (p. 64). Benveniste a très vivement le sens de cette clôture de l'un à l'autre, dans l'ordre du savoir.

La systématicité de la pensée du système déduit du premier principe de non redondance un second principe: "Il n'y a pas de signe transsystématique » (p. 53). Prenant sur la phrase précédente : "La valeur d'un signe se définit seulement dans le système qui l'intègre». A l'exemple que prend Benveniste, du rouge des feux de circulation au rouge du drapeau

\footnotetext{
5 En fait, implicitement, d'avoir écrit que « la langue est l'interprétant de la société », contient au moins les paradigmes social et politique, mais je n'ai pas connaissance que Benveniste ait développé ce problème.
} 


\section{Henri Meschonnic}

tricolore, on pourrait ajouter le noir du deuil, et l'erreur d'un critique de plaquer ce noir sur les noirs des peintures de Soulages, confusion entre une valeur culturelle d'une couleur et des valeurs qui sont seulement celles d'une oeuvre particulière.

Dans tout cela, Benveniste s'occupe essentiellement de caractériser le fonctionnement de la langue, de «système interprété » à "système interprétant $»$ (p. 54).

La confrontation entre la musique et la langue mène à illustrer qu'il y a de l' «inconvertible» (p. 5 5). Benveniste le dit de la musique à la langue. Il va de soi que c'est réciproque, de la langue à la musique. Et qu'il en va de même pour les autres arts : peinture, cinéma, etc. Chaque fois spécifiquement.

En musique, l'inconvertible vaut non seulement pour les notes, qui ne sont pas des signes, mais aussi pour le rapport au temps: "l'axe des simultanéités en musique contredit le principe même du paradigmatique en langue, qui est principe de sélection, excluant toute simultanéité intrasegmentale » (p. 56). D'où : « la combinatoire musicale qui relève de l'harmonie et du contrepoint n'a pas d'équivalent dans la langue» (p. 56). Benveniste conclut, dans l'hypothèse où « la musique est considérée comme une 'langue', que c'est une langue qui a une syntaxe, mais pas de sémiotique». Où il n'emploie pas du tout le terme de langue selon la métaphore courante du langage musical, qui présuppose exactement le contraire, et par cette présupposition même à la fois procure l'illusion de penser et empêche de penser un rapport entre la musique et le langage. C'est au sens précisément linguistique, ici, qu'intervient la langue, comparée à la musique. Donc la musique n'a pas de mots. Par quoi elle n'a pas le sens. Ce qui ne signifie pas qu'elle n'a pas de sens. Mais qui ouvre justement ici un jeu de langage, aussi traditionnel que non maîtrisé.

Il faut ajouter que Benveniste ne cherche visiblement pas ici à penser la musique, mais seulement à «éclairer la sémiologie de la langue» (p. 56). Cependant l'exemple des arts plastiques va renforcer la notion selon laquelle des systèmes pourraient même ne pas avoir d'unités. La question même si la couleur, le trait sont des unités y perd son sens.

Une première conséquence de cette réflexion est une distinction à retenir entre art et oeuvre, que Benveniste ne souligne pas, momentanément, mais qui compte, et qu'il fait entrer plus loin (p. 59) dans sa démonstration : «Aucun des arts plastiques considérés dans leur ensemble ne paraît reproduire un tel modèle» (p. 56) - le modèle qui ne s'observe complètement que dans la langue, et qui comporte «1) un répertoire fini de signes, 2) des règles d'arrangement qui en gouvernent les figures 3 ) indépendamment de la nature et du nombre des discours que le système permet de produire » (p. 56). C'est là que Benveniste note : « Tout au plus pourrait-on en trouver quelque approximation dans l'oeuvre de tel artiste; il ne s'agirait plus alors de conditions générales et constantes, mais d'une caractéristique individuelle, et cela encore nous éloignerait de la 
langue » (p. 57). Ce que reprend, plus loin (p. 65), et systématise, l'expression: « sémantique (expressions artistiques), sans sémiotique ».

En quoi il y a un nominalisme de l'oeuvre (tout autre que celui des mots), qui s'oppose au réalisme (au sens logique) de l'art: «L'art n'est jamais ici qu'une oeuvre d'art particulière » (p.59). Un ici qui ne désigne sans doute que le rôle et la place de l'argument, non une atténuation de sa portée théorique. A prendre cette proposition absolument, elle met à vif le conflit entre l'esthétique et la poétique.

A ce stade, la notion d'unité « est au centre de la problématique qui nous occupe » (p. 57 ).

La grande nouveauté de Benveniste, ici, est d'oser dissocier unité et signe. La proposition: "Le signe est nécessairement une unité, mais l'unité peut n'être pas un signe » (p. 57) est capitale pour les «expressions artistiques ». Si l'oeuvre tout entière est l'unité, pour la poétique, l'unité n'est pas un signe, et l'oeuvre n'est pas faite de signes. Une oeuvre de langage est pleine de mots, mais ce n'est pas les mots qui font l'oeuvre, c'est l'oeuvre qui fait ce qu'on attribue ensuite aux mots. Ce n'est pas en tant quelle est composée de signes quelle est une oeuvre. Ce que déjà Humboldt disait du discours en général, que les mots ne le précèdent pas mais qu'ils en procèdent, est d'autant plus vrai d'une oeuvre de langage, comme système de discours.

Autrement dit, dans une oeuvre de langage, Benveniste aurait pu dire : «L'artiste crée ainsi sa propre sémantique » au lieu qu'il a écrit : «L'artiste crée ainsi sa propre sémiotique » (p. 58. Puisqu'il disait de l'artiste: "Il ne reçoit donc pas un répertoire de signes, reconnus tels, et il n'en établit pas un " (p. 58).

Cette sémiotique limite est annulée par là-même. Une sémiotique d'une seule unité ne peut pas être une sémiotique, puisqu'il n'y a de sémiotique sinon d'un système de signes, généralisable et généralisé à d'autres signes, à d'autres discours, comme c'est le cas dans une langue. Elle est en cours de constitution dans une oeuvre, surtout les oeuvres de longue durée, celle de Hugo par exemple. Elle a ses effets de traîne chez les épigones. Pas pour longtemps, elles ne traînent que chez les contemporains. Cette sémiotique à un seul exemplaire (sa reproduction étant le corollaire même de la négation d'une oeuvre), Benveniste ne la dit ainsi peut-être que par un reste d'opposition binaire, que l'oeuvre se charge d'exténuer : l'artiste "institue ses oppositions en traits qu'il rend lui-même signifiants dans leur ordre » (p. 58). Mais «signifiants dans leur ordre » conviendrait aussi bien, et mieux même, à du sémantique. Comme Benveniste lui-même le dit, en trois mots, plus loin.

Dans tout cela, il ne s'agit que de penser une critique du langage. Non d'abaisser les problèmes à de la polémique. Il s'agit de situer Benveniste dans cette critique de la pensée du langage. Et par là-même nous situer aussi. Il ne 
s'agit pas de faire l'apologie de Benveniste ${ }^{6}$. Mais seulement un exercice de méthode. Un travail. En vue de quoi il n'y a pas à se soucier d'imputations qui semblent avoir d'autres valeurs que celles du travail.

Il s'agit de chercher ce qui est fécond dans Benveniste, et partout, ce qui permet de penser le langage non en reproduisant Benveniste, mais à partir de lui. Ce qui suppose, dans ce à-partir-de, qu'il y a, avec Benveniste, une oeuvre, telle qu'on ne peut plus faire comme si elle n'avait pas eu lieu. La définition d'une oeuvre, ici dans ses effets, c'est qu'on ne peut ni faire comme avant, ni la refaire.

La définition même du classique. Celle qu'en donne Reinhart Koselleck dans une conférence sur Goethe ${ }^{7}$, en reprenant des mots de Karl Löwith, à propos de Goethe, cette définition du classique étant: "Impossible d'en revenir à lui, mais tout autant impossible de passer par-dessus lui - Unmöglich, zu ihm zurückzukehren, aber ebenso unmöglich, über ihn hinauszukommen ».

C'est son élaboration du discours qui fait de Benveniste un chemin obligé (ce qui dit bien qu'on ne s'arrête pas à lui) pour penser le continu dans le langage, une position linguistique du sujet dans son énonciation qui prépare, mais ne constitue pas, la notion de sujet du poème ${ }^{8}$; notion rhétorique, poétique, éthique et politique du sujet, et par là, intempestivement aussi contre la sémiotisation générale ambiante, une pensée de l'historicité et de la valeur telle que penser la poétique et penser la modernité deviennent une seule pensée.

Ce cheminement même dégage une modernité de Benveniste. Un cas de plus, pour le comique de la pensée, où la modernité, que certains croient finie, survit et survivra au post-moderne.

En quoi il y a bien aussi un style de la linguistique, parce que la pensée est affaire de style, et que même elle est tout le style. Plus proche en cela de l'art que de la science, sinon que dans la science même il y a ceux qui ont du style, et ceux qui n'en ont pas. L'invention, où tient l'allure, et qui est l'historicité même.

Mais le post-moderne chez un certain nombre de linguistes, tout comme chez des spécialistes de littérature, ou des philosophes, participe des formes diverses d'éclectisme qui déshistoricisent les concepts, les désituent - croyance naïve à leur efficacité hors de leur système - et se croient d'autant plus d'aujourd'hui qu'elles repsychologisent, et rebiologisent, à contre-Saussure.

Ici le post-moderne se ramasse précisément dans l'oubli de ces trois mots de Benveniste : "sémantique sans sémiotique ». Parce que le post-moderne est le côté de Peirce.

\footnotetext{
${ }^{6}$ Comme il m'a été dit à propos de l'article «Seul comme Benveniste ou Comment la critique manque de style ».

7 Reinhart Koselleck, «Goethes unzeitgemässe Geschichte » [L'histoire intempestive de Goethe], Collegium Budapest, Public Lectures, n'6, January 1994, p. 2.

8 Abordée dans Politique du rythme, politique du sujet.
} 
Il s'agit donc de partir des trois mots de Benveniste, avec l'incise entre parenthèses, "expressions artistiques", au pluriel, son extension à tous les arts. De considérer ces trois mots comme un programme, avec ses problèmes. Des prolongements que sans doute ne prévoyait pas Benveniste. Mais il les a rendus nécessaires.

Dans le contexte où il énonçait cette propriété, selon lui, des "expressions artistiques", il s'agissait de balancer deux types opposés de systèmes dans la langue, et à la langue.

Selon ses propres termes, une "signifiance unidimensionnelle: ou sémiotique (gestes de politesse, mudras), sans sémantique; ou sémantique (expressions artistiques), sans sémiotique ». La double signifiance de la langue, celle « des signes » et celle « de l'énonciation », permettant, selon Benveniste, la «faculté métalinguistique ", la «relation d'interprétance par laquelle la langue englobe les autres systèmes » (p.65). Deux extrêmes, ou limites, puisque, communément, la langue est bi-dimensionnelle, sémiotique et sémantique.

Une mesure même de la force de ce passage est dans les obscurités qu'il contient. Car ces deux catégories, malgré leur symétrie inverse apparemment parfaite, n'appellent pas du tout la même analyse. Et il n'en reste pas du tout la même chose.

De la première catégorie (dont je n'ai pas vu, jusqu'ici, de critique, faute peut-être d'information) je dirais qu'elle n'est pertinente qu'à se contenter d'apparences.

Un coup de chapeau, un geste de politesse justifient, mais tout juste, la catégorie comme telle. Dès qu'on prend ces gestes davantage dans leur jeu social, il est de plus en plus difficile d'en exclure une sémantique.

Les mudras sont les "poses ritualisées et stylisées" des mains des Bouddhas et des Bodhisatvas (bodhitsatva est celui dont l'essence est bodhi, « l'éveil», et qui " retarde sa propre entrée dans le Nirvana pour travailler au salut de tous les êtres conscients ») 9 . Par rapport aux poses des jambes, asana. Ces poses ont chacune un sens spécifique, codé. Elles ne sont un exemple du sémiotique sans sémantique, et dans sa perfection, que vu de l'extérieur du code, par ceux qui l'ignorent. Mais chaque pose, vue par des initiés, a un sens. L'encyclopédie iconographique, que je viens de citer, les traite comme des symboles. Il y a donc une sémantique des mudras.

Quant au jeu social courant, et variable, des gestes de politesse, on ne peut pas non plus soutenir, du fait même qu'ils concernent des postures du corps, qu'il soient dénués d'une sémantique.

Comme on peut voir, en caricature, dans certains récits humoristiques de Tchékhov. Concrètement, par exemple, dans Le gros et le maigre (Tolstyj $i$ tonkij), de 1883. Deux personnages qui ne se sont pas vus depuis l'enfance se retrouvent à la sortie d'un train. Ils sont devenus fonctionnaires. Le maigre

9 Dans An Encyclopaedia of Budhist Deities, Demigods, Godlings, Saints \& Demons, prof. Fredrick W. Bunce, New-Delhi, Printworld Ltd, 1994, t. 2, p. 1019. 


\section{Henri Meschonnic}

(l'administration tsariste comporte quatorze grades, ou rangs) est au huitième rang, rang moyen, assesseur de collège (kollezhskij asessor). Dès qu'il se rend compte que l'autre, le gros, est, non pas comme il supposait d'abord, à le voir, du cinquième rang, conseiller d'État (statskij), mais du troisième rang, conseiller privé (tajnyj - le mot comporte une idée de secret et de mystère), c'est l'équivalent de "lieutenant général », il change complètement de langage, et de gestuelle, forcément, car les deux vont ensemble.

Aussitôt il donne au gros du « votre excellence » (vache prevoskhoditel'stvo) qui ne se dit que pour les officiels du troisième rang (et vache-stvo, dans une autre nouvelle, Mort d'un fonctionnaire), avec des suffixes archaïsants de respect qui s'ajoutent aux mots. Il ne dit plus «c'est très agréable " (otchen 'prijatno) mais otchen'prijatno-s), avec un -s, qui s'ajoutait dans l'ancienne Russie à n'importe quel mot, en finale de groupe (initiale de "seigneur », sudar, pour gosudar).

Rien de corporel n'est oublié. Leur odeur. Le gros sent le Xéres et la fleur d'oranger ; le maigre, le jambon et le café. Leur encombrement : le gros est seul, le maigre est flanqué d'une femme, derrière lui, et d'un fils lycéen, qui se cache derrière le père quand celui-ci le présente. Le volume sonore : le gros crie en voyant l'autre. Ils ont tous deux les yeux pleins de larmes. L'onomatopée des rires. Le gros fait Ho-ho. A l'annonce du grade du gros, le maigre pâlit, il se pétrifie. Son rire se hiérarchise. Ce n'est plus Hi-hi, c'est Khi-khi-s. Ce qui rend le gros malade : « Il se détourna du maigre et lui donna pour un adieu la main. / Le maigre serra trois doigts, s'inclina de tout son corps, et eut de petits rires, comme un Chinois : 'Hi-Hi-Hi'».

L'exemple de ce récit de Tchékhov suffit à illustrer que les signes de politesse sont à la fois du corps individuel et du corps social, en même temps qu'ils sont liés au registre de langue, au sens de la stylistique de Bally en 1909. Les signes de politesse étant l'expression de relations sociales portent donc aussi une sémantique. Ils ne sont pas du sémiotique sans sémantique. Ils appartiennent au «monde de l'énonciation », à «l'univers du discours» (p. 64). Il y a en eux du reconnu, mais aussi du compris. Dans le récit de Tchékhov, le comique de la chute sociale s'exprime dans l'affaissement du petit devant celui qu'il met si haut. La hiérarchie est dans le corps et dans le langage.

Mais même les gestes de politesse ordinaire ne sont pas dénués de sémantique, et d'implicite. Sous-entendus variables, liés aux effets d'intonation qui les accompagnent. Même sans paroles il y a des inflexions gestuelles. Puisque les gestes sont le corps social. La catégorie du "sémiotique sans sémantique ", pour les gestes de politesse, ne concerne peut-être qu'un nombre de signaux stéréotypés restreint à l'état pur. Ce qui dans cette mesure même, limite cette catégorie, au point de ne lui donner qu'une existence tout abstraite.

Pour le corps, sauf erreur, Benveniste n'en parle pas. Je dirais que c'est le corps qui contraint, ou ne contraint pas, le langage à inventer du dire. Le corps transforme le langage, ou ne le transforme pas. Je dis le corps, je veux dire la physique du plaisir ou de la douleur, la physique du sentir et du comprendre faisant ou ne faisant pas la physique du langage. Et c'est là spécifiquement 
l'activité de l'art, et l'activité des oeuvres. Le passage même du problème de Wittgenstein (l'inadéquation radicale de dire j'ai mal à la douleur, que seul le gémissement porte, mais il est hors langage) au problème d'Adorno (qu'il est impossible d'écrire après Auschwitz, parce qu'il est impossible d'écrire Auschwitz). C'était déjà le problème de Brice Parain, dans Petite métaphysique de la parole ${ }^{10}$. Le problème classique de l'hétérogénéité radicale entre le continu de la vie et le discontinu du langage (aussi chez Bergson). L'anthropologie du signe. Incapable de dire l'émotion, c'est-à-dire incapable d'un continu entre le corps et le langage, et, pour la même raison, incapable d'expliquer que ce continu existe, et que c'est la littérature. La même carence théorique devant la vie et devant le langage.

Problème inclus, mais non posé, quand Benveniste dit : « le langage sert à vivre » (Il, p. 217). Or ce problème passe par donner du corps au langage. Il commence précisément dans les trois mots sémantique sans sémiotique.

La symétrie que présente Benveniste entre les deux systèmes à «signifiance unidimensionnelle » est toute d'apparence. En réalité, derrière la réversibilité formelle des termes, autant la catégorie du sémiotique sans sémantique est ténue et de principe, pour ne pas dire vide, autant celle du sémantique sans sémiotique ouvre un problème sans mesure commune avec son symétrique inverse, et est seule à l'ouvrir.

Portant expressément et uniquement sur les "expressions artistiques ", l'infinité des expressions artistiques, cette catégorie se trouve aussi sans commune mesure avec les autres activités de langage, puisqu'elles sont toutes bidimensionnelles.

Benveniste venait d'établir que dans la musique «l'unité peut n'être pas un signe» (p.57) et que dans «les arts de la figuration (peinture, dessin, sculpture)", c'est aussi «l'existence même d'unités qui devient matière à discussion » (p. 58). Il disait : «S'il s'agit de couleurs [... ] elles sont désignées, elles ne désignent pas ». C'est là que Benveniste neutralisait l'opposition entre sémiotique et sémantique, en écrivant que «l'artiste crée ainsi sa propre sémiotique ».

Ce qu'il développait alors: «Les relations signifiantes du 'langage' artistique sont à découvrir A L'INTERIEUR d'une composition. L'art n'est

\footnotetext{
10 Gallimard, 1969, p. 20-21 : « [...] c'est la vie, qu'on ne comprend pas. / Il me semble néanmoins que ce n'est pas le même mouvement qui me fait regarder longuement la mer, que je suis venu voir de loin, et dire ce que je peux en dire après. La suite naturelle de la contemplation serait le silence. Sous le coup de cette puissance gigantesque, qui m'attire et m'effraie à la fois, il me faudrait quelque temps pour m'en délivrer, pour ne plus me sentir écrasé, vaincu, fasciné. Je suis là, sur la rive, à ne rien entreprendre pour affronter l'Océan. [... ] Il m'arrive pourtant, en lisant, de trouver dans un livre des moments qui ressemblent à ce que j'ai pu éprouver. Je me dis : celui-là (l'auteur) a su employer les mots qui me parlent. Il y a sûrement une correspondance entre le langage et le réel. Seulement laquelle? Il a pu écrire des phrases émouvantes, mais ce n'est pas nécessairement parce que son émotion s'est dite elle-même dans ses paroles. Sinon pourquoi la mienne ne produirait-elle pas des poèmes, elle aussi ? Parce qu'elle ne serait pas assez forte, ou parce qu'elle le serait trop, au contraire ? Elle me ferme plutôt la bouche. J'en reste ahuri, interloqué, muet ».
} 


\section{Henri Meschonnic}

jamais ici qu'une oeuvre d'art particulière, où l'artiste instaure librement des oppositions et des valeurs dont il joue en toute souveraineté, n'ayant ni de 'réponse' à attendre, ni de contradiction à éliminer, mais seulement une vision à exprimer, selon des critères, conscients ou non, dont la composition entière porte témoignage et devient manifestation » (p. 59).

Où le rapport entre sémiotique et sémantique devient interne à l'oeuvre, et du coup un critère de ce qu'on peut appeler une oeuvre. L'oeuvre est toujours "particulière» si elle est à la fois sa sémantique et sa sémiotique. Autre manière de dire finalement que sémiotique et sémantique sont une seule et la même - tension à la Héraclite - et qui révèle la tension interne au sémantique sans sémiotique.

Mais cela ne se vérifie qu'à condition d'y inclure - ce que Benveniste a laissé implicite, et impensé - une distinction introduite par la philosophie analytique entre emploi descriptif neutre de la notion d'oeuvre, et emploi évaluatif ${ }^{11}$. C'est-à-dire à condition de prendre la notion d'oeuvre dans son sens implicitement évaluatif réalisant le degré maximal de sa propre définition : au sens où une oeuvre n'est vraiment une oeuvre que si elle accomplit au maximum ce que fait une oeuvre, ce qui fait que quelque chose est une oeuvre, et non pas quelque chose qui ressemble à une oeuvre. Inclusion de la valeur dans la définition.

Quant à la peinture, il y a lieu d'ajouter que la notion de couleur, que prélève Benveniste, n'a pas de sens isolément, car, dans une " oeuvre d'art particulière », elle est toujours inséparable d'une matière, d'une texture, d'une forme (et des formes qui l'entourent, représentatives ou non), et d'une manière, comme l'analyse Gérard Dessons. La couleur est une abstraction. Parler de couleur montre involontairement la distance entre le langage (ou du moins ce discours) et la peinture, qui est toujours telle ou telle peinture.

Un autre détail reste énigmatique : ce que pouvait entendre Benveniste par les "arts de la figure ", après les "arts de la figuration », qui désignent clairement la peinture, puisque Benveniste y parle des couleurs que l'artiste «dispose à son gré sur la toile» (p. 58). Il ajoutait: «Quant aux arts de la figure, ils relèvent déjà d'un autre niveau, celui de la représentation, où trait, couleur, mouvement se combinent et entrent dans des ensembles gouvernés par des nécessités propres » (p. 59). Peut-être qu'il s'agit du figuratif...

En tout cas, il n'y a bien sûr pas lieu de restreindre à la peinture ce que Benveniste donne alors comme conclusion partielle, par sa phrase : «L'art n'est jamais ici qu'une oeuvre d'art particulière ». Puisque c'est toute oeuvre, si elle est, une, oeuvre, qui est "particulière ", quelle que soit sa matière.

Il s'y ramasse donc, sans que Benveniste ait développé ce problème, la question du sujet de l'oeuvre, sujet du poème, sujet spécifique, qui n'est pas le

\footnotetext{
11 Comme le montre, après Goodman, Charles Stevenson dans l'article «Qu'est-ce qu'un poème ? » (de 1957, traduit dans Poétique, n'83 , septembre 1990, et repris par Gérard Genette dans son recueil Esthétique et poétique, Seuil, 1992).
} 
sujet de l'énonciation courante ; la question de son historicité ; la question de sa modernité. Toutes trois formant le problème de la poétique.

Benveniste caractérise le particulier de l'oeuvre d'art dans les termes apparents du discours traditionnel sur la littérature, auteur, monde clos. Les oeuvres sont «les systèmes où la signifiance est imprimée par l'auteur à l'oeuvre ", à la différence de ceux "où la signifiance est exprimée par les éléments premiers à l'état isolé »; les systèmes " où la signifiance se dégage des relations qui organisent un monde clos», par rapport à ceux où elle est «inhérente aux signes eux-mêmes». La signifiance dans l'art est telle qu'elle «ne renvoie donc jamais à une convention identiquement reçue entre partenaires » (p. 59).

Or les termes qu'emploie ici Benveniste ont ceci de remarquable qu'ils font système contre la lecture structuraliste qui prédomine alors, et dont un effet retard est qu'aujourd'hui encore Benveniste est vu par certains comme un structuraliste $^{12}$. Il est intéressant de confronter les termes de Benveniste avec ceux de Michel Foucault, exactement contemporains de "Sémiologie de la langue », dans "Qu'est-ce qu'un auteur ? » Foucault priait Lucien Goldmann de lui épargner «les facilités sur le structuralisme». Contre la vulgate illusionnée de la mort du sujet, il redonnait une vigueur théorique au terme d'auteur, il le renforçait même, et le rapportait spécifiquement à celui de sujet, mais en ajoutant: "Encore faudrait-il préciser dans quel champ le sujet est sujet, et de quoi (du discours, du désir, du processus économique, etc.). Il n'y a pas de sujet absolu ${ }^{13}$. Ce qui devrait contribuer à la critique de l'expression vulgate la-question-du-sujet.

Le terme de "systèmes ", ici en rapport avec " auteur » et « oeuvre », et le rejet de la "convention ", liée à la chaîne du conventionnalisme, en même temps que toute la corrélation entre philologie et linguistique générale, et l'attitude déductive, situent Benveniste hors du structuralisme. La vulgate structuraliste confond système et structure, elle dit structure, et non système. Et elle éliminait l'auteur, confondu avec le sujet.

Benveniste n'est pas structuraliste. Mais il n'est pas pour autant dans le psychologisme littéraire traditionnel, puisqu'il pose que l'oeuvre d'art est du sémantique sans sémiotique. Par quoi, sans le savoir, Benveniste met l'oeuvre dans le continu du rythme héraclitéen, "configurations particulières du mouvant » (I, p. 333). Alors que nulle part, dans les textes que nous connaissons de lui, il ne met en rapport, pour la poétique, son article sur le rythme et l'oeuvre, sémantique sans sémiotique.

La situation de Benveniste a ici un double intérêt: partant de l'oeuvre d'art particulière, elle implique une critique de la vulgate structuraliste et une critique de la vulgate sémiotique, dont elle montre l'alliance et les limites.

\footnotetext{
12 J'ai montré, dans «Seul comme Benveniste, ou Comment la critique manque de style », qu'il n'en était rien.

13 Michel Foucault, Dits et écrits, Gallimard, 1995, t. 1, p. 818.
} 
Henri Meschonnic

C'est ce qu'illustre aussi une longue note qui rassemble ses objections contre une sémiologie de l'art, en distinguant entre le "répertoire iconique » des "thèmes religieux», qui désigne une sémiotique de la culture, et «le véritable problème sémiologique, qui à notre connaissance n'a pas encore été posé», et qui "serait de rechercher COMMENT s'effectue cette transposition d'une énonciation verbale en une représentation iconique » (p.59). Tout étant dans le comment, qui est la question même de la poétique.

Le rejet de la notion de convention (« une convention identiquement reçue entre partenaires ») indique ce que la théorie a pour tâche de dégager : l'implication réciproque entre la poétique d'une oeuvre d'art particulière (et elles sont toutes particulières) et la poétique de la poétique - qui est la théorie générale du langage. La situation unique dans le langage du sémantique sans sémiotique définit à la fois l'art et la littérature, et la théorie du langage comme critique du signe.

Il ne s'agit pas d'une critique technique du signe. Ou plutôt il ne s'agit pas seulement d'une critique de la technologisation du signe qu'a été la vulgate structuraliste. Il s'agit, plus largement, d'une critique de la perte de théorie qu'a été la vulgate structuraliste. Ce qui est comique, si on se rappelle que pour beaucoup le structuralisme se confondait avec un abus de la théorie - par confusion du formalisme avec la théorie. Je dis perte de théorie pour désigner la perte du rapport entre rhétorique, poétique, éthique et politique; pour désigner la perte du continu dans la représentation seule du discontinu; le substitut d'une pensée du continu - d'une poétique - étant, pour beaucoup, le déconstructionnisme, qui a pris la polysémie pour critère de littérarité, et qui montre par là combien il reste pris dans les schémas du structuralisme littéraire.

C'est sur une telle critique, inévitablement, mais sans la formuler, qu'ouvre, à propos de "la signifiance de l'art», la phrase: "Il faut en découvrir chaque fois les termes, qui sont illimités en nombre, imprévisibles en nature, donc à réinventer pour chaque oeuvre, bref inaptes à se fixer en une institution » (p. 59-60).

Or, dans l'énoncé de Benveniste se rencontrent deux problèmes et une caractéristique forte.

Le premier problème intervient à propos de la « relation d'interprétance » (p. 61), qui a lieu "entre un système interprétant et un système interprété » (p. 61). Cette relation définit la langue comme un "rapport sémiologique" distinct $\mathrm{du}$ « rapport sociologique». Elle fait que «seule la langue permet la société » (p. 62), et que «c'est la langue qui contient la société ». Non l'inverse.

Le problème est celui de la possibilité et des limites du rapport entre la relation d'interprétance et la relation d'homologie.

Benveniste avait distingué trois «types de relation»: la relation d'engendrement, comme de l'écriture alphabétique à l'alphabet Braille, ellemême distincte du "rapport de dérivation", par exemple «entre l'écriture hiéroglyphique et l'écriture démotique» (p. 61); la relation d'homologie, 
«instaurée en vertu de connexions qu'on découvre ou qu'on établit entre deux systèmes distincts », comme les correspondances de Baudelaire, ou celles « que Panofsky voit entre l'architecture gothique et la pensée scolastique »; enfin la « relation d'interprétance ", rapport propre à la langue, et qui fait de la langue «l'interprétant de tous les systèmes sémiotiques» (p.61). Y compris non langagiers. Où le langage est le métalangage. L'interprétant, mais comment et jusqu'où?

Ce problème apparaît si on confronte le premier trait de la relation d'interprétance avec la relation d'homologie. Le premier trait est « l'énonciation, qui porte référence à une situation donnée ; parler, c'est toujours parler-de ».

Mais Benveniste n'a pas évoqué le double fond, si je peux dire, de l'expression parler-de. Le plus communément du monde, parler-de pose un objet externe à l'acte d'énonciation, un objet devant un sujet, un objet dont il est dit quelque chose, dans la relation classique entre thème et prédicat. Y compris quand cet objet est le sujet lui-même. C'est le sens un.

Or on ne peut pas oublier, quand cette fonction est confrontée à l'oeuvre d'art, précisément quand celle-ci est définie - reconnue - comme une sémantique sans sémiotique, que le parler-de se trouve dans une relation d'extériorité radicalement insatisfaisante, par rapport à une transitivité interne que désigne non plus parler-de, mais dire. En quoi il faut désormais distinguer entre un transitif externe, pour dire, quand il s'agit du mode bidimensionnel du langage (dire quelque chose), et un transitif interne, quand il s'agit du sémantique sans sémiotique, pour une oeuvre d'art. La relation alors n'est plus d'interprétance, mais d'une homologie spécifique. Dire et faire y sont un et le même ; forme, particulière à l'oeuvre d'art, du performatif. Métaphore, si on veut, du performatif linguistique. Mais juste à titre transitoire.

Ce qu'il s'agit de caractériser, c'est que, s'il y a « sémantique (expressions artistiques), sans sémiotique » (p. 65) dans une oeuvre de langage, quelle que soit la diversité infinie de sa réalisation, le sémantique à la fois dit et fait, dans le continu d'un système de discours, une activité spécifique radicalement distincte du parler-de, et transformatrice de ce qu'il dit, transformatrice du mode de signifier, de comprendre, d'écrire et de lire. Ce que ne peut capter ni une lecture thématique, qui le réduit à des contenus de vérité, ni une lecture des formes.

Par rapport au sens neutre de parler-de, contrasté à ce dire, parler-de se charge d'une valeur d'insuffisance, péjorative et secondaire. Sens second, sens marqué. Le sémantique seul met en difficulté le parler-de, qui est une fonction du signe.

L'oeuvre ne saurait parler-de, à moins d'annuler la spécificité qui a été postulée pour elle, et qui permet de la reconnaître. Parler-de n'est pas dire, comme un poème, à sa façon, fait ce qu'il dit. Et ne parle pas de. S'il parle de, ce n'est pas un poème, mais du simili. Je dis poème emblématiquement. C'est tout autant spécifique des grands romans.

Benveniste ne fait pas cette distinction. Il est donc amené à une contradiction cachée : son analyse a un point faible et un point fort. Le point 


\section{Henri Meschonnic}

faible est qu'il ne semble pas penser cette difficulté, cette spécificité. Le point fort est que l'expression de sémantique sans sémiotique prépare au problème, et nous oblige à le penser.

C'est dans ce conflit même, et seulement à partir de la reconnaissance de ce conflit entre dire et parler-de, et ici le sens second rejoint le sens premier de parler-de, c'est entre une irréductibilité de l'oeuvre d'art particulière au parlerde et le parler de, que se situe la relation entre la poétique et la théorie du langage.

S'il y a une oeuvre faite d'un autre élément que le langage (musique, peinture, sculpture, cinéma - où le langage entre en composition, mais n'est pas ce qui fait le cinéma, etc.), le parler-de se heurte à une difficulté autre et la même, mais d'autant plus forte qu'elle est rendue inapparente par le langage même, fondue dans sa fonction générale, le parler-de au sens premier. Dans le signe absence des choses. Un parler-de qui ne saura jamais dire un faire qui, cette fois, lui est hétérogène, et on retombe au sens second.

On peut donc légitimement se demander - comme on fait depuis longtemps - ce que peut dire d'une oeuvre d'art particulière la relation d'interprétance, autrement dit ce qui reste du sémantique-sans-sémiotique dans la "métasémantique » postulée par Benveniste à la fin de son article, comme " analyse translinguistique des textes, des oeuvres » (p. 66); comment il faudrait que ce parler-de soit conçu (ou, à son tour, comment il devrait transformer la théorie générale du langage) pour comprendre un dire et un faire qui n'ont rien d'un parler-de. N'ayant pas de sémiotique.

C'est tout le conflit entre le sémantique sans sémiotique et la langue, puisque la langue est ce parler-de en tant quelle est du sémiotique "à la fois dans sa structure formelle et dans son fonctionnement » (p. 62).

Un deuxième problème, qui dépend du premier, apparaît quand Benveniste écrit que la langue est «produite et reçue dans les mêmes valeurs de référence chez tous les membres d'une communauté » (p. 62). Ce qui est bien sûr la définition même du caractère social de la langue. Or il venait de dire, à propos de la signifiance de l'art, qu'«il faut en découvrir chaque fois les termes, qui sont illimités en nombre, imprévisibles en nature, donc à réinventer pour chaque oeuvre, bref inaptes à se fixer en une institution » (p. 59-60).

Il y a donc immédiatement un heurt entre l'infini du sens et de la valeur propres à l'oeuvre, toujours susceptibles d'une lecture nouvelle, l'infini sémantique de l'oeuvre, diversité, altérité (toujours si c'est une oeuvre) et la totalité sémiotique de la langue, qui suppose un primat de l'identité : «les mêmes valeurs de référence ». C'est-à-dire la culture, ses traditions, son lieu et son moment. Avec ses effets de stase, dus aux pouvoirs.

Là encore, le conflit entre un infini sémantique et une totalité sémiotique est une donnée immédiate et nécessaire des rapports entre la poétique et la théorie du langage. La question est : comment la relation entre une sémantique sans sémiotique et la langue comme système sémiotique-sémantique peut-elle être elle-même sémiotique? Car Benveniste dit bien que «pour déterminer les 
relations entre systèmes sémiotiques, nous avons posé que ces relations doivent être elles-mêmes de nature sémiotique » (p. 62).

Ce n'est pas là prendre Benveniste en défaut, mais rien d'autre que tenter de penser les problèmes du langage. Tâcher de mettre au jour, c'est-à-dire aussi mettre à vif les contradictions inhérentes au rapport même entre le tout sémiotique de la langue et la sémantique sans sémiotique des oeuvres d'art.

Cette double difficulté, du faire confronté au parler-de, et du multiple imprévisible de la valeur confronté à l'identité-totalité (elle-même variable) de la langue, est montrée et cachée dans l'exposé de Benveniste. Il me semble qu'elle donne la démonstration même que le rapport entre la sémantique sans sémiotique et la sémiotique-langue ne tombe pas juste et ne tombera jamais juste: il y a et il y aura toujours un reste, indéfini et infini, qui échappe à l'interprétance, et qui définit un avenir irréductible de la valeur et du sens, dont le lieu est l'art, la difficulté de penser l'art, et que, de l'art, il n'y a jamais que des oeuvres. Il n'y a que le particulier pour penser le général!

Cette situation fait que les moments de sémantique sans sémiotique sont seuls la chance d'une liberté et d'un avenir du sujet dans et par le sujet de l'art, le sujet du poème. Par là le sujet du poème est une liberté pour les autres sujets et, son avenir étant son présent continué, ce sujet est le sujet même de la modernité, comme activité permanente du présent.

Autant, quand il y a sémantique sans sémiotique, cesse la double signifiance propre à la relation d'interprétance de la langue, et qui en fait un «modèle sans analogue » (p.63), autant cesse alors la double articulation du langage. Car l'articulation en monèmes et en phonèmes, décrite par Martinet, n'est pertinente que dans les limites où le langage est pensé dans les termes de la langue, qui sont les termes du discontinu traditionnel entre les mots, entre les phrases, entre le son et le sens, ou la forme et le sens.

Mais cette représentation, qui n'est pas une nature des choses, bien quelle se donne pour telle, mais seulement une représentation, perd toute pertinence hors de ses limites, qui sont celles de la langue. Dès que le langage est considéré dans l'ordre du discours, dans la physique du discours (et non plus seulement selon le logicisme de la pragmatique), et spécialement dans des systèmes de discours qui sont seuls l'accomplissement maximal du sémantique sans sémiotique, ce qui s'observe est le fonctionnement du continu, que masque la représentation du signe.

Le continu est le rythme comme organisation du mouvement de la parole dans l'écriture, et l'oralité non plus comme l'opposition duelle de l'oral à l'écrit dans le signe, mais comme le primat du rythme et de la prosodie dans le mode de signifier. Des sémantiques sérielles, des "prosodies personnelles", disait Apollinaire. Comme organisatrices d'un récit second on peut les appeler un récitatif. Il y a du sémantique sans sémiotique quand le continu du sujet du poème fait l'historicité d'un texte. Quand le récitatif fait que son récit domine le récit de l'énoncé. Alors, bien plus que du sens et des formes, il y a une activité 
Henri Meschonnic

du discours, à écouter dans ce qu'elle fait, qui n'est pas nécessairement ce que disent les mots.

Que le sémantique sans sémiotique ne puisse avoir lieu que dans le discours, non dans la langue, est préparé par l'analyse même du discours, chez Benveniste, en termes de globalisation, non d'identités successives : "ce n'est pas une addition de signes qui produit le sens, c'est au contraire le sens (l"intenté'), conçu globalement, qui se réalise et se divise en 'signes' particuliers, qui sont les MOTS » (p. 64).

Conception remarquablement proche d'une formule fameuse de Humboldt: "Dans la réalité, le discours n'est pas composé de mots qui le précèdent, mais ce sont les mots au contraire qui procèdent du tout du discours » ${ }^{14}$. Il y a là un penser-Humboldt, qui retire tout sens au rapprochement, par le terme d'« intenté », entre Benveniste et Husserl - rapprochement fréquent dans la tradition structuraliste, particulièrement chez Julia Kristeva. Car Benveniste pense le discours, non les mots, et Husserl ne connaît que les noms : « ... fehlen uns die Namen ", comme il écrit à la fin du \$36 des Leçons sur la phénoménologie de la conscience intime du temps.

Ce qui s'oppose aux notions d'unité et d'identité dans le discontinu du signe, où prévaut la double articulation, n'est pas l'absence d'unité et d'identité, mais l'interaction et l'implication réciproque des éléments du discours dans le continu.

Le problème de la poétique, et le problème poétique, n'est pas seulement qu'il y a «deux univers conceptuels » (p. 64), mais qu'il y a entre eux un conflit. Ce que Benveniste ne dit pas. Il évoque seulement leur dissociation dans les «formes pathologiques du langage» (p. 65). Mais il a tout fait pour qu'on soit contraint de l'affronter. Il ne s'agit plus de l'opposition entre «reconnaître » et « comprendre » (p. 65), mais d'une crise du comprendre. C'est la caractéristique forte de ce texte.

Cette crise n'est pas nouvelle. Elle est même constitutive de l'histoire des représentations du langage et de la poésie. Mais Benveniste la pousse à un paroxysme, avec son « sémantique (expressions artistiques), sans sémiotique ». Pour la pousser, afin qu'elle en vienne à une rupture, qui est nécessaire, il faut dire, selon une absurdité seulement apparente, que le sémantique sans sémiotique n'est pas de la langue, ne participe pas de «l'univers conceptuel» du signe. Ni même vraiment du discours tel que Benveniste en a formé le concept. Le paradoxe supplémentaire est que c'est le langage même, parce qu'il est communément représenté par le discontinu du signe, qui cache le fonctionnement asémiotique du langage.

C'est pourquoi le «deuxième niveau d'énonciation » (p. 65), comme dit Benveniste, "cette faculté métalinguistique», ne suffit plus, telle qu'elle est

\footnotetext{
14 «In der Wirklichheit wird die Rede nicht aus ihr vorangegangenen Wörtern zusammengesetzt, sondern die Wörter gehen umgekehrt aus dem Ganzen der Rede hervor », W. von Humboldt, éd. Cotta, III, p. 448 ; éd. de l'Académie, VII, p. 72 ; éd. du Seuil, Introduction à l'œuvre sur le Kavi, p. 213.
} 
située, pour analyser cette sorte d'énonciation particulière à «l'oeuvre d'art particulière » (p. 59). Il y faut une transformation générale des représentations du langage : une critique du signe par une poétique du rythme.

Pour cela, c'est le programme inachevé de Benveniste qui est à reprendre, quand, pour le "domaine sémantique », il requérait : "Il aura besoin d'un appareil nouveau de concepts et de définitions» (p. 65). C'était pour penser le discours, au sens où le discours était le domaine indifférencié du comprendre. C'était encore le discours-de-la-langue, puisque «la langue comporte deux domaines distincts, dont chacun demande son appareil conceptuel» (p. 65).

Mais le sémantique sans sémiotique est un empêcheur de penser par deux : de penser dans le dualisme. Il est un peu oublié, ici. Or il demande réellement "un appareil nouveau de concepts et de définitions", qui est appelé nécessairement à modifier la représentation commune, celle du discours, où il s'insère, et celle du signe, le récit que se raconte le signe de sa propre histoire. Dans son sommeil dogmatique.

Il reste, aujourd'hui non seulement à continuer, comme Benveniste a commencé, de penser le discours avec des concepts du discours, pour ne pas reglisser du discours à la langue, en psychologisant, en logicisant, il reste à penser une sémantique sérielle du continu. Il reste à penser le rythme comme rhétorique, poétique, éthique et politique du sujet.

Avec le problème de l'effet de théorie que peut avoir cette critique du rythme, cette poétique de l'énonciation, sur la linguistique de l'énonciation.

Apparemment, c'est une dialectique à la hégélienne que Benveniste propose, à la fin de "Sémiologie de la langue ", pour trouver une issue au blocage de la théorie du langage par le conflit entre le discours et le signe: «On ne pouvait écarter l'idée du signe linguistique sans supprimer le caractère le plus important de la langue ; on ne pouvait non plus l'étendre au discours entier sans contredire sa définition comme unité minimale. / En conclusion, il faut dépasser la notion saussurienne du signe comme principe unique, dont dépendraient à la fois la structure et le fonctionnement de la langue » (p. 66).

En fait, seul le mot dépasser donne l'apparence d'une démarche hégélienne. Benveniste ne fait pas un dépassement, mais un déplacement notionnel, par l'apport de concepts nouveaux. L'ensemble reste du côté de Saussure, mais s'oppose au structuralisme. C'est un premier effet de théorie de "Sémiologie de la langue». Puisque, mieux aujourd'hui on peut connaître Saussure, philologiquement, mieux on voit combien la vulgate structuraliste avait altéré les concepts de Saussure. Mais le rapport d'interaction entre langue et parole (transformé en couple d'exclusion mutuelle par les structuralistes), non seulement n'est pas dépassé, mais il est relayé, repris, par le concept de discours.

Ce que Benveniste voyait comme un dépassement était dit presque entièrement au futur, et annoncé comme un mouvement double: "Ce 


\section{Henri Meschonnic}

dépassement se fera par deux voies ». Et si la première voie ne fait pas difficulté, dans la seconde est tout le problème.

La première voie résume la bi-partition fondamentale, à l'intérieur de la langue. C'est ainsi que je comprends l'intralinguistique: "dans l'analyse intralinguistique, par l'ouverture d'une nouvelle dimension de signifiance, celle du discours, que nous appelons sémantique, désormais distincte de celle qui est liée au signe, et qui sera sémiotique ». La part concernant le discours est au présent, car le concept est acquis. Ce qui concerne le sémiotique est au futur.

Car le travail sur les notions déplace les limites du concept de langue, et de tous les concepts qui en dépendent. Ce travail ne se fait pas sans un doublon sur le terme de discours, qui a été noté par Gérard Dessons, dans la double opposition de langue à discours et de discours à récit. Mais il s'accompagne d'un porte-à-faux, par un changement de sens, du coup, sur langue, par rapport à la vulgate structuraliste.

Toute la deuxième voie est au futur: "dans l'analyse translinguistique des textes, des oeuvres, par l'élaboration d'une métasémantique qui se construira sur la sémantique de l'énonciation. / Ce sera une sémiologie de 'deuxième génération', dont les instruments et la méthode pourront aussi concourir au développement des autres branches de la sémiologie générale ». Fin du texte de Benveniste.

C'est sur cette voie que je situe la poétique du rythme. Elle est seulement annoncée par Benveniste comme un avenir de la théorie. Je n'ai pas vu, du moins dans les textes qu'on connaît de lui, qu'il y soit revenu. En 1969, son oeuvre s'arrêtant, "Sémiologie de la langue » reste un programme futur. Mais, comme faisait Saussure, ce qui n'est pas encore découvert est prévu, sa place est là.

Cependant, l'« appareil nouveau de concepts et de définitions » n'est pas là. Il est encore presque entièrement inconnu.

Quant à l'analyse «translinguistique » - si « intralinguistique » désigne bien, dans la langue, les deux domaines du sémiotique et du sémantique - il me semble qu'elle désigne une sortie hors de la langue, donc hors du signe, par le sémantique sans sémiotique, puisqu'il s'y agit « des textes, des oeuvres ».

Rien n'est dit, et ne pouvait l'être alors davantage, de cette «métasémantique», sinon quelle sera celle des oeuvres. Elle est le travail même de ce que j'appelle la poétique, et qui n'a que le nom de commun avec telle ou telle stratégie formelle, néo-rhétorique des figures ou continuation de Roman Jakobson.

Quelque chose de l'état théorique antérieur - l'état commun du signe semble bien demeurer dans l'évocation même de l'utopie théorique, par le terme de "sémiologie ". Il conserve la notion de Saussure au moment même où Benveniste déclare qu'«il faut dépasser la notion saussurienne du signe comme principe unique ». Sans doute pour conserver le projet de Saussure d'une sémiologie générale. 
La «sémiologie de la langue » y jouerait le rôle principal. Puisque ses « instruments » et sa «méthode » pourront « concourir au développement des autres branches de la sémiologie générale ». Projet bien situé par ce moment du structuralisme, dans les années 60-70, où la linguistique était vue comme la science pilote, «le patron général de toute sémiologie » écrivait Saussure, que citait (p. 49) Benveniste.

Aujourd'hui si on regarde ce projet, ce qui est curieux c'est que, contrairement à ceux - les plus nombreux - qui croient que la linguistique n'a plus ce rôle, qu'elle a été remplacée dans ce rôle de pilote par la sociologie de Bourdieu, il n'en reste pas moins, et même plus que jamais, que c'est la théorie $\mathrm{du}$ langage qui est la visée majeure des tentatives pour penser une théorie d'ensemble de la société, une anthropologie historique du langage, et du sujet. C'est ce que montrent, avec des succès, ou plutôt des insuccès divers, les entreprises philosophiques et sociologiques de Habermas et de Bourdieu.

Après le structuralisme linguistique, c'est un avenir de Saussure, par le rôle de la théorie du langage - l'expression est de lui - dans une théorie critique de l'art et de la société. L'avenir même de la modernité après le post-modeme.

Dans ses incertitudes, et cet emploi du futur, la fin de «Sémiologie de la langue " n'est pas une fin, mais l'inaccompli de la théorie répondant à l'inaccompli qui est le mode même d'activité des oeuvres. Ce qui fait qu'elles continuent. Que seules les vraies continuent. Dans leur poétique prend la poétique. Le sémantique sans sémiotique est cette double poétique, celle de l'activité des oeuvres et l'activité de les reconnaître, de les connaître.

Par sa difficulté même, cette formule est un "noeud rythmique", comme disait Mallarmé. Elle implique encore une autre question, qui retrouve celle du rapport entre interprétance et homologie : si la pensée du sémantique, et même, plus généralement, si la pensée du langage n'est pas, chez les inventeurs de cette pensée, à la différence des ingénieurs, plus proche de l'oeuvre d'art que de la science. Ou tiendrait des deux. Avec du continu entre les deux, plus que leur opposition banale. Non comme la dénégation du métalangage, chez Barthes, mais comme métalangage. Ainsi la pensée du sémantique sans sémiotique réaliserait cette contradiction, d'être à la fois une «métasémantique » et une relation d'homologie.

Il n'y a là, après tout, que des effets de représentation, dans ces cloisons supposées. Mais il est certain qu'il y a plus à chercher, et à trouver, dans les incertitudes du sémantique sans sémiotique, que dans les certitudes du signe.

Pour moi, bien qu'il y ait plus d'incertitudes que de certitudes dans la poétique, je ne parlerais plus de "sémiologie générale", car c'est encore le signe, mais de théorie du langage, puisque, par une sorte d'homologie, comme "la langue est l'interprétant de la société », la théorie du langage est l'interprétant de la théorie de la société. Et le sémantique sans sémiotique, critère de l'art, et du sujet du poème, est lui-même le critère du rôle de l'art et de la littérature comme critère des représentations de la société. 
Henri Meschonnic

C'est pourquoi je mettrais du côté du sémantique sans sémiotique le début de La Poétique d'Aristote, que j'ai placé comme un monument à l'inconnu, au commencement de Politique du rythme, politique du sujet, depuis qu'une relecture, bonheur ou erreur, mais lectio difficilior, m'a fait voir le titre composé non plus des deux premiers mots, mais des trois premiers, Sur la poétique même (Peri poiêtikês autès) et non plus Sur la poétique, comme le voulait la tradition, puisque la suite énonce que son objet, l'objet de la poétique même n'est pas tant les vers ou la prose, ni la différence entre les genres, que «ce qui se trouve sans nom jusqu'à maintenant » (anônumos tunkhanei ousa mekhri tou nûn), et cette analogie, venue de loin, ignorée jusqu'ici, cette solidarité entre la poétique et le sémantique sans sémiotique, aussi actuelle aujourd'hui qu'il y a si longtemps, fait leur passé et leur avenir. 